\title{
Photometry of the low accretion rate polar HS 1023+3900
}

\author{
R. Schwarz ${ }^{\star}$ A. D. Schwope , and A. Staude \\ Astrophysikalisches Institut Potsdam, An der Sternwarte 16, 14482 Potsdam, Germany \\ Received 12 March 2001 / Accepted 9 May 2001

\begin{abstract}
We present results from a photometric monitoring campaign of the new low $\dot{M}$ magnetic CV HS 1023+3900. It showed the system at a constant brightness level during the half year of observations, confirming that it is permanently accreting at a low rate. Optical light curves are dominated by emission from two cyclotron spots in accordance with previously published spectroscopy. The timings of the primary maxima were used to derive a revised spin-period of $166.93 \mathrm{~min}$. We estimated approximate locations of the accretion spots on the white dwarf by simple modeling of the $R$ and $I$-band light curves. Interestingly, the dipole axis is perpendicular to the line connecting both stars and inclined into the orbital plane, an orientation quite different from that found in all other polars so far. We report the detection of a single flare event of $\Delta B \sim 1$ mag lasting $\sim 1 \mathrm{~min}$, indicating chromospheric activity of the secondary.
\end{abstract}

Key words. accretion - stars: cataclysmic variables - AM Herculis binaries - stars: individual: HS $1023+0039$ stars: magnetic fields

\section{Introduction}

The magnetic cataclysmic variable (mCV) HS 1023+3900, hereafter referred to as HS 1023, was recently discovered by Reimers et al. (1997) from the Hamburg Quasar Survey. Contrary to most CVs it was not recognized by its strong $\mathrm{X}$-ray radiation nor its line emission spectrum, but rather by a single cyclotron line superimposed on the spectrum of the late-type companion. Follow-up spectroscopy revealed two cyclotron systems from different accretion regions having different magnetic field strengths of about $60 \mathrm{MG}$ and $68 \mathrm{MG}$. The total flux measured in cyclotron lines was consistent with an accretion rate of only $\sim 10^{-13} M_{\odot} / \mathrm{yr}$, two orders of magnitude lower than usually found in CVs at this period. With this unique property it appears to be the first object of this class in a permanent state of reduced accretion.

The absence of disturbing effects of accretion makes HS 1023 itself an ideal laboratory to study the white dwarf's magnetic field (via cyclotron and Zeeman spectroscopy) and the status of the secondary.

Furthermore it might be a key object for the understanding of cataclysmic binary evolution as a whole. With an orbital period of 2.78 hours it is well located in the

Send offprint requests to: R. Schwarz, e-mail: rschwarz@aip.de

* Visiting astronomer, German-Spanish Astronomical Center, Calar Alto, operated by the Max-Planck-Institut für Astronomie, Heidelberg, jointly with the Spanish National Commission for Astronomy.

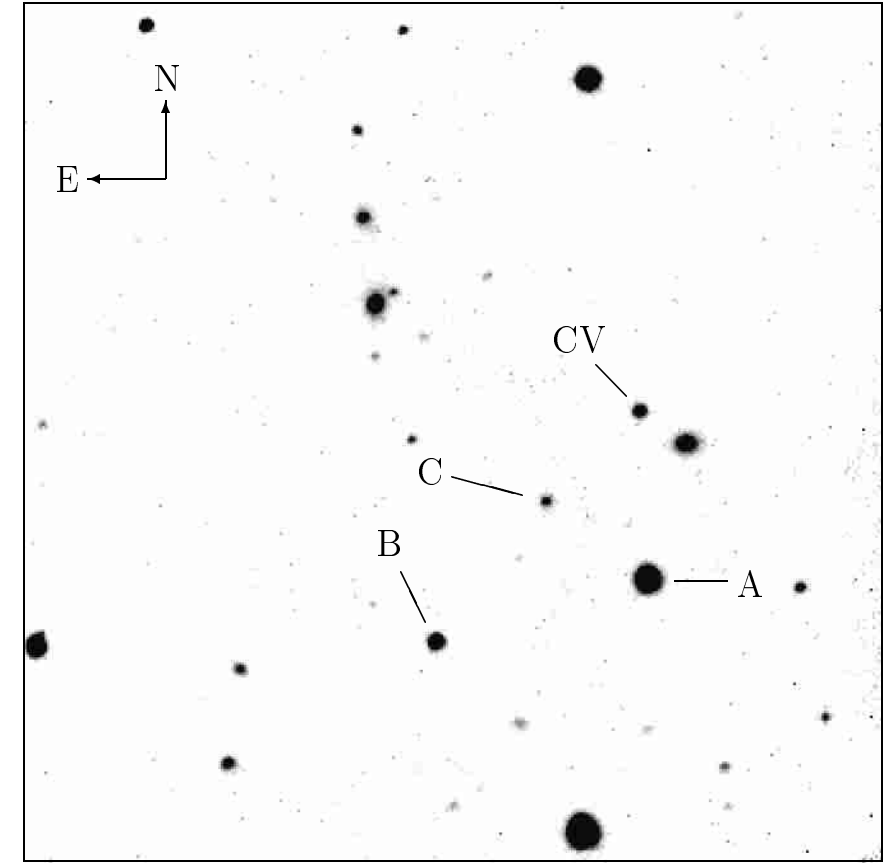

Fig. 1. V-band CCD image of HS 1023 (labelled as "CV") obtained with the AIP $70 \mathrm{~cm}$ telescope. North is on top and east to the left. The size of the field is approximately $8 \times 8 \mathrm{arcmin}^{2}$.

period gap of CVs, a range of periods between 2 and 3 hours in which substantially fewer CVs are observed. This lack of systems implies either an ultra-fast evolution through the gap, or an evolution in a detached state. 
Table 1. Log of optical observations of HS 1023.

\begin{tabular}{llllrll}
\hline Telescope $^{a}$ & Date & Instrument & Filter & $\#^{b}$ & $\begin{array}{c}\text { Duration } \\
(\mathrm{h})\end{array}$ & $\begin{array}{l}\Delta T_{\text {int }} \\
(\mathrm{s})\end{array}$ \\
\hline AIP 0.7-m & 10 Dec. 98 & TEK & $V$ & 179 & 3.41 & 120 \\
AIP 0.7-m & 20 Jan. 99 & TEK & $B V R$ & 117 & 4.96 & $60,30,30$ \\
DSAZ 3.5-m & 10 Mar. 99 & TWIN & $B R V$ & 50 & 5.02 & 300 \\
AIP 0.7-m & 17 May 99 & TEK & $B V I$ & 100 & 4.38 & $60,30,30$ \\
AIP 0.7-m & 19 May 99 & TEK & $V R I$ & 48 & 2.68 & $40,30,30$ \\
AIP 0.7-m & 26 May 99 & TEK & $V$ & 82 & 3.52 & 120 \\
AIP 0.7-m & 27 May 99 & TEK & $R I$ & 83 & 3.25 & 60,60 \\
\hline
\end{tabular}

${ }^{a}$ AIP: Astrophysical Institut Potsdam, DSAZ: German-Spanish Astronomical Center.

${ }^{b}$ Optical: number of exposures.

The widely accepted working hypothesis for the formation of the gap favours the latter, and ascribes this to a sudden stop of "magnetic braking", which operates at periods longer than 3 hours.

Thus HS 1023 might be in one of two possible evolutionary states. First, it may be a pre-CV just at the onset of accretion, or secondly and even more intriguing, it had been already accreting as a normal CV above the gap, and is now detached by the mechanisms which are responsible for the creation of the gap.

So far, photometry of HS 1023 comprised only ten hours, and extended only over a baseline of 10 days. In this paper, we describe the results of a photometric monitoring campaign, which primarily aimed to obtain a better understanding of its long-term behavior and a more accurate photometric period.

\section{Observations}

We observed HS 1023 during six nights between December 1998 and May 1999 with the $0.7 \mathrm{~m}$ reflector of the Astrophysical Institute Potsdam, Germany. Observations were done with $B V$ and $R I$ filters in the Johnson and Cousins system, respectively. For some observations the filters were subsequently changed during nights to provide quasi-simultaneous colour information. Images were taken with a $1024^{2}$ TEK CCD detector with integration times ranging between $30-120 \mathrm{~s}$. Their automated reduction included standard calibration (bias, flat-field), transformation of image offsets and the computation of profile-fitting magnitudes using the DoPHOT (Mateo \& Schechter 1989) package. Differential magnitudes of HS 1023 were derived with respect to star "A" marked in Fig. 1. A complete log of the observations is given in Table 1 .

We additionally extracted broad-band light curves from phase-resolved medium-resolution spectroscopy obtained on March 81999 with the $3.5 \mathrm{~m}$ telescope at Calar Alto. This was done by folding these data with filter transmission curves representing standard filters.

On March 181999 the field of HS 1023 was observed together with a set of Landoldt standard stars
(Landoldt 1992) with MOSCA at the Calar Alto $3.5 \mathrm{~m}$ through $U B V R I$ filters. From that, we derived calibrated magnitudes (see Table 2) of stars A, B and C marked in Fig. 1.

Table 2. Magnitudes of comparison stars. The values for HS 1023 correspond to the phase range $\phi_{\text {phot }}=0.06 \ldots 0.13$ of our photometric ephemeris (Eq. (1)).

\begin{tabular}{lccccc}
\hline Star & $U$ & $B$ & $V$ & $R$ & $I$ \\
HS & 17.49 & 18.04 & 16.97 & 16.40 & 14.83 \\
A & 16.77 & 15.59 & 14.43 & 13.60 & 12.88 \\
B & 17.70 & 17.26 & 16.38 & 15.76 & 15.16 \\
C & 20.48 & 19.76 & 18.35 & 17.26 & 15.80 \\
\hline
\end{tabular}

\section{Optical variability}

\subsection{The spin period}

The broad-band photometry of HS 1023, as illustrated by the quasi-simultaneous $B V R$ light curves observed on January 201999 (Fig. 2), can be readily understood in the context of the changing visibility of two cyclotron systems spectroscopically observed by Reimers et al. (1997).

This is most obvious in the $R$-band, where we observe a primary maximum of $\Delta R \sim 0.5$ mag caused by the $60 \mathrm{MG}$ pole (hereafter referred to as the primary pole), which is followed about half an orbit later by a secondary maximum of $\Delta R \sim 0.25 \mathrm{mag}$, when the $68 \mathrm{MG}$ pole (hereafter referred to as the secondary pole) is visible. Cyclotron emission in the $R$-band is due to the 3rd harmonic (at $5950 \AA$ ) of the primary pole and the 2nd harmonic (at $7700 \AA$ ) of the secondary pole. In the $V$-band the primary maximum is due to the same $3 \mathrm{rd}$ harmonic of the $60 \mathrm{MG}$ pole as in the $R$ band, while the secondary pole emits only in the higher 3rd harmonic in that bandpass. Contrary to all other harmonics, this one shows strong cyclotron beaming (Reimers et al. 1997). Emissivity of that harmonic is highest when our line of sight is perpendicular to magnetic 
HS1023+39

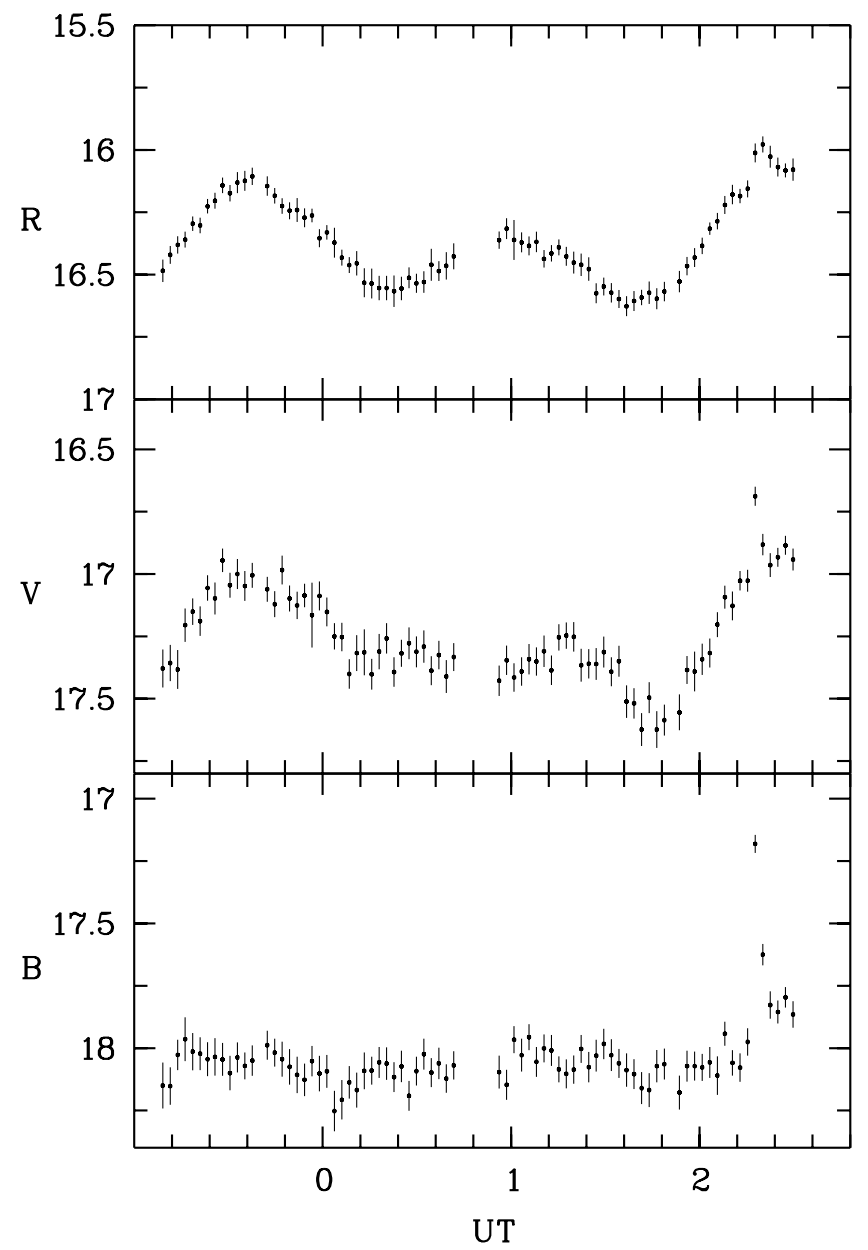

Fig. 2. Multi-colour $B V R$ light curves observed quasisimultaneously on January 201999 . Note the $\sim 1$ mag flare in the $B$-band occuring at UT 2:18.

field lines in the spot, i.e. when the secondary pole rotates over the limb of the white dwarf into and out of view. This results in a double-humped secondary maximum observed in our $V$-band light curve at UT 0:24 and UT 1:18.

The $B$-band is apparently devoid of emission from higher harmonics, and the corresponding light curve is therefore constant except for a 1 mag flare event during the end of the observation. Any residual modulation apart from the flare, e.g. caused by higher cyclotron harmonics, is less than $0.1 \mathrm{mag}$. In none of the colours do we observe the flickering behavior reported by Reimers et al. (1997).

We applied the analysis-of-variance method (Schwarzenberg-Cerny 1989) to the $V$ and $R$ data for a period search. While both data sets lead to the detection of many alias periods, caused by the sparse sampling, there is only one period of $166.93 \mathrm{~min}$ consistently detected in both data sets (Fig. 3). This new period differs by $\sim 40 \mathrm{~s}$ from that derived by Reimers et al. (1997), and should preferentially used for any further study.

We also determined individual mid-times of the primary maxima by fitting cosines to the data excluding the

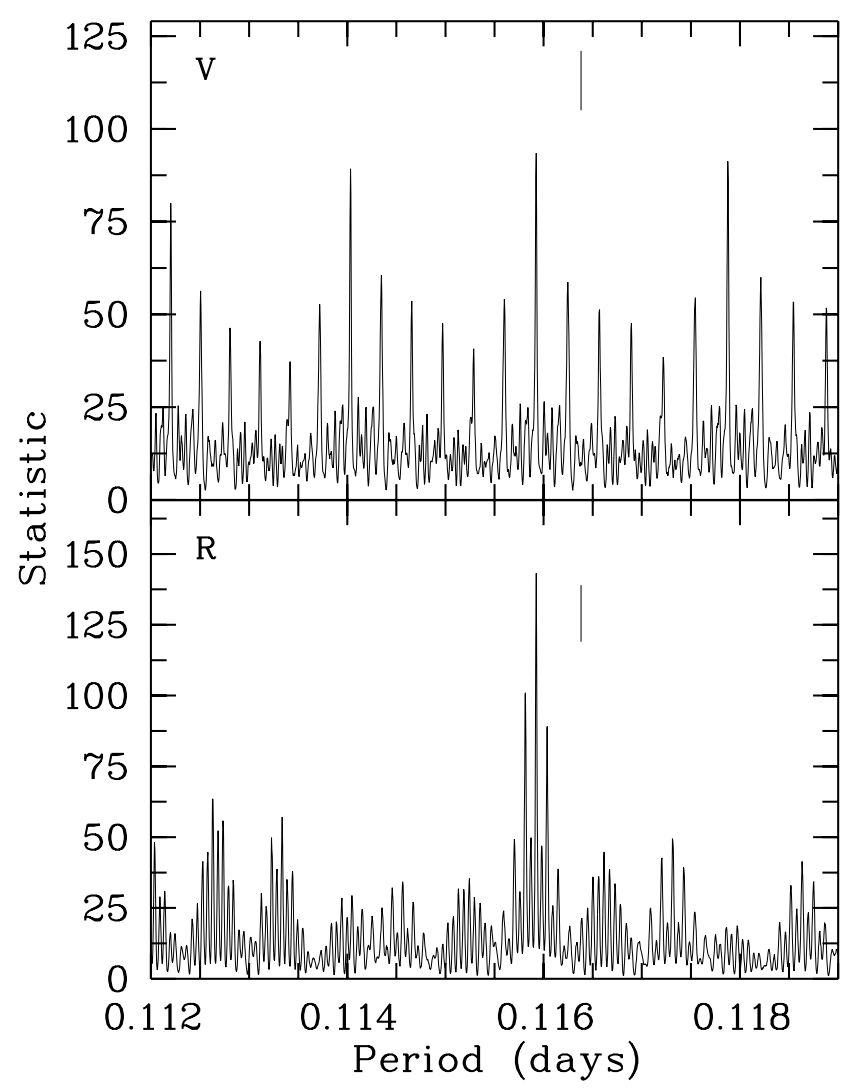

Fig. 3. Periodograms derived from optical $V$ and $R$ photometry using the analysis-of-variance method (SchwarzenbergCerny 1989). Likely periods appear as maxima. The only period consistent with both data sets is at 166.93 min. The ticks mark the period from Reimers et al. (1997).

secondary hump (see Table 3). A weighted linear regression of all $V$-band maxima, which has the longest baseline of all filters used by us, yields the photometric ephemeris,

$\operatorname{HJD}\left(T_{\max }\right)=2451157.6439(9)+E \times 0.1159229(13)$

where the numbers in brackets represent the $1 \sigma$ uncertainties in the last digits. The residuals of the linear fit (observed minus calculated times of the photometric maxima) are shown in Fig. 4 . The scatter of the $V$-band timings of about $\Delta \phi \sim 0.05$ is within the formal errors of the cosine fit. For the quasi-simultaneous measurement, we also note that minima observed in the $I$-band occur about $\Delta \phi \sim 0.05$ earlier than the timings in the $R$ or $V$-band.

\subsection{Long term behavior}

During the half year of observations HS 1023 displayed only a change of the overall intensity at a level of few per cent. HS 1023 was observed with an orbital mean brightness $R=16^{\mathrm{m}} 33$ through the observing season with a standard deviation of only $0.02 \mathrm{mag}$, just at the limit of the photometric accuracy of our observations. An additional measure is the amplitude of the primary maximum which was $\Delta R=0.61 \mathrm{mag}$ and varied only by $0.05 \mathrm{mag}$. 
Table 3. Heliocentric timings of the primary photometric maxima.

\begin{tabular}{|c|c|c|c|c|}
\hline $\begin{array}{c}T_{\max } \\
(\text { HJD } 2400000+)\end{array}$ & $\begin{array}{l}\Delta T_{\max }^{a} \\
\left(10^{-4}\right.\end{array}$ & $\begin{array}{l}\mathrm{O}-\mathrm{C} \\
\mathrm{d})\end{array}$ & Cycle & Filter \\
\hline 51157.64431 & 11 & 4 & 0 & V \\
\hline 51199.48881 & 8 & -32 & 361 & $R$ \\
\hline 51199.49069 & 13 & -13 & 361 & $V$ \\
\hline 51248.53359 & 33 & 62 & 784 & $V$ \\
\hline 51316.45624 & 9 & -20 & 1370 & $R$ \\
\hline 51316.45225 & 13 & -59 & 1370 & $I$ \\
\hline 51318.42806 & 16 & -8 & 1387 & $V$ \\
\hline 51318.42385 & 12 & -50 & 1387 & $I$ \\
\hline 51325.50630 & 55 & 61 & 1448 & $V$ \\
\hline 51326.54449 & 102 & 10 & 1457 & $R$ \\
\hline 51326.54234 & 91 & -12 & 1457 & $I$ \\
\hline
\end{tabular}

${ }^{a}$ Errors resulting from cosine fit.

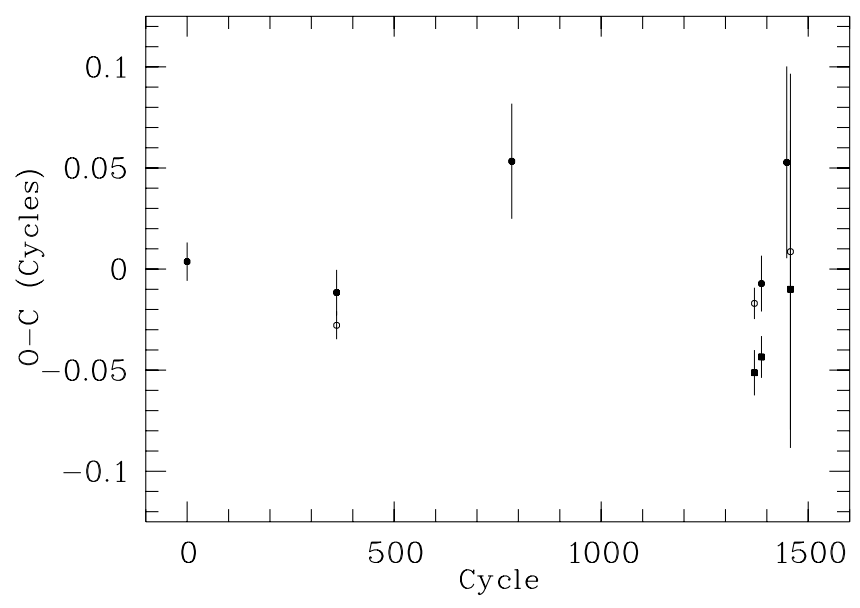

Fig. 4. Diagram of observed minus calculated times of the photometric primary maxima computed with respect to the linear ephemeris of Eq. (1). Different filters used, are indicated by squares $(I)$, open $(R)$ and filled circles $(V)$.

\subsection{The flare}

On January 201999 we observed a short flare of at least 0.95 and $0.35 \mathrm{mag}$ in $B$ and $V$, respectively. With a time resolution of about $40 \mathrm{~s}$, the rise to maximum was not measured and the decay only barely resolved. When first detected in $V$ it was already declining in the $B$-band. From that, we estimate a rise time of less than $40 \mathrm{~s}$ and a substantially longer decay time of $120 \mathrm{~s}$. In the $B$-band a peak flux of $5 \times 10^{-16} \mathrm{erg} \mathrm{cm}^{-2} \mathrm{~s}^{-1}$ was reached. The $(B-V)$ colour of $-0.01 \mathrm{mag}$ of the flare is much bluer than that of the cyclotron spots. Instead, the colour and time signature of that event are very similar to UV Ceti flare stars (Moffett 1974) and we conclude that we observed a flare on the secondary and not an accretion flare.

\section{Colours and magnitudes of the stellar components}

For a short period at around $\phi_{\text {phot }}=0.7$, both accretion poles are behind the limb of the white dwarf and out of view for the observer. Thus the magnitudes and colours observed during that phase can be used to estimate the contribution of the stellar components, the white dwarf's temperature, the secondary's spectral type and the distance of system.

Assuming that for $\lambda<5000 \AA$ the red dwarf contributes only a negligible fraction to the total luminosity, the observed colour $U-B=-0.58$ is consistent with a $13000 \mathrm{~K}$ hot white dwarf in agreement with the spectroscopic estimate of Reimers et al. (1997).

After subtracting the white dwarf's flux contribution, we find colours of $R-I=1.82$ and $V-R=1.73$ for the red companion. These can be compared with colours of late, single main sequence stars (Kirkpatrick \& McCarthy 1994) and would imply spectral types of M 4.5 and M 5.5, respectively. This inconsistency is within the limits set by our photometric accuracy of $0.1 \mathrm{mag}$, but might also indicate a wrong extrapolation of the white dwarfs flux at longer wavelengths. Since the $R-I$ colour is less prone to these effects we adopt a spectral type of M 4.5 for the secondary in the following.

With an observed visual magnitude of $I=15.11$ and the absolute magnitude of a dM 4.5 star $(I=10.18)$ the implied distance of HS 1023 is $\sim 100$ pc, slightly lower but still in agreement with Reimers estimate.

\section{Simple modeling of the light curves}

Fortunately, both accretion columns in HS 1023 are obscured by the primary for part of the orbit. The duration of these self-eclipses provides constraints on the system geometry, e.g. the inclination $i$ and the spot colatitudes $\beta$. Simple geometric considerations lead to

$\cos \gamma \pi=\cot i \cot \beta$

where $\gamma$ is the fraction of the orbit for which the spots are invisible.

In the following we used phase-averaged light curves (Fig. 5) computed from the complete data set mainly to improve the signal/noise. These have a phase resolution of 0.02 and an average error of 0.02 mag. As a first step we determined $\gamma$ by measuring the fraction of the orbit for which the spot brightness exceeds the off-phase level at $\phi_{\text {phot }}=0.7$. For the primary spot we find almost identical values in the $R$ and $I$-band of $\gamma_{1, R}=0.537$ and $\gamma_{1, I}=0.548$, which put it on the hemisphere far from the observer. For the secondary spot we find the same value $\gamma_{2, R}=0.537$ and both spots should be located at roughly the same colatitude. Compared to the $R$-band, the duration of the faint phase is notably shorter $\left(\gamma_{2, I}=0.495\right)$ in the $I$-band.

So far, the only constraint on the inclination is the absence of eclipses, setting $i \lesssim 72^{\circ}$. This considerably 


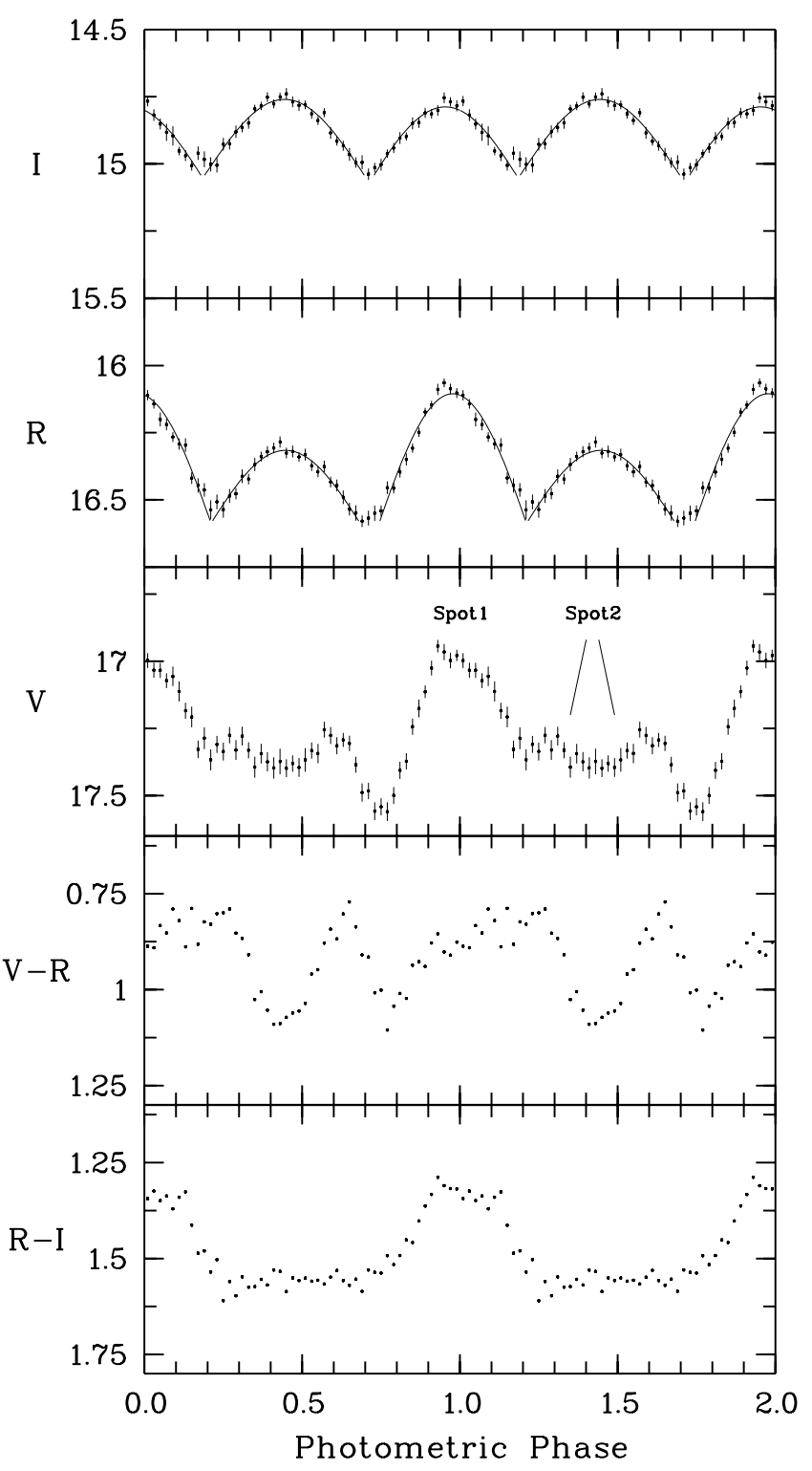

Fig. 5. Binned and phase-averaged $V R I$ light curves using all available data and corresponding $V-R$ and $R-I$ colour as a function of photometric phase. Solid curves are results from fitting simple foreshortening of two spots.

narrows the possible range of spot colatitudes given by Eq. (2) to $90^{\circ} \lesssim \beta_{1,2} \lesssim 110^{\circ}$, or, if we use the $\gamma_{2, I}$ for the secondary spot in the $I$-band, to $80^{\circ} \lesssim \beta_{2} \lesssim 90^{\circ}$. Thus the magnetic axis is likely to be inclined into the orbital plane.

The cosine shape of the lower harmonics in the $R$ and $I$-band suggests that cyclotron emission is not strongly beamed (as seen in the 3rd harmonic of the secondary pole) but comes from an optically thick region. Brightness changes are then due to the foreshortening of the spot and proportional to the cosine of the viewing angle $\alpha$, where $\cos \alpha$ is related to the inclination $i$, spot azimuth $\psi$ and colatitude $\beta$ by the cosine rule. We could therefore model the light curves fitting the $\cos \alpha$ function using a $\chi^{2}$ minimization routine.

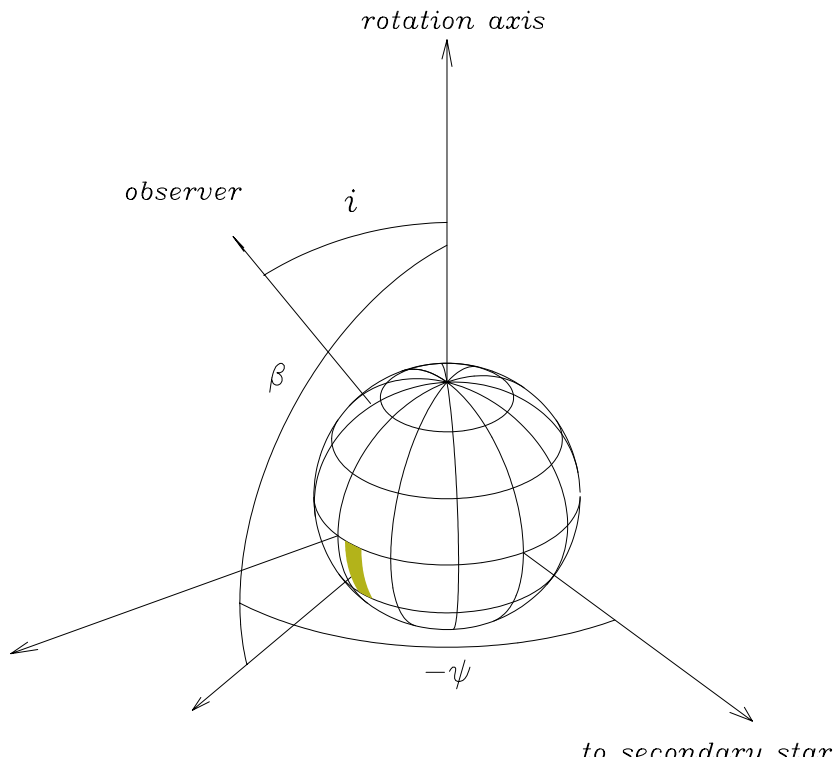

primary spot

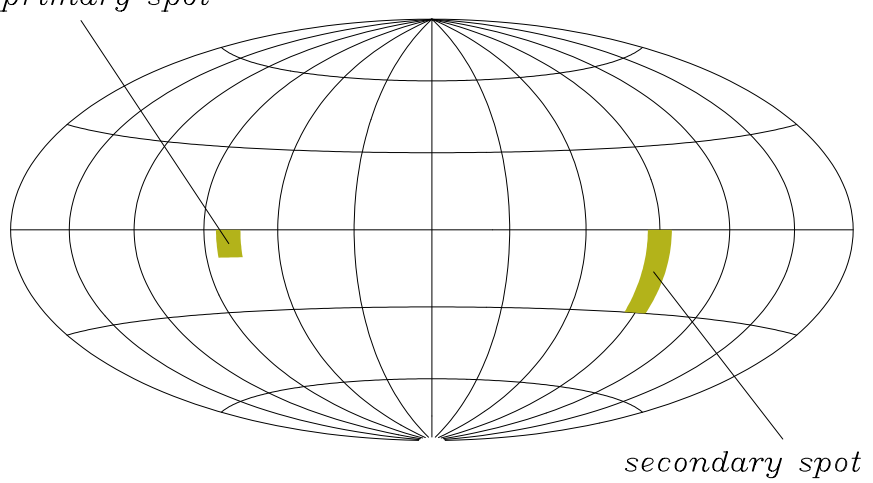

Fig. 6. Top: principle geometry of a white dwarf in a magnetic CV observed at an inclination $i$ with the angles $\beta$ (colatitude) and $\psi$ (azimuth) defining the location of the accretion spot. Bottom: map of the white dwarf's surface in HS 1023 showing the likely positions of the primary and secondary accretion spot as derived is this work. Central meridian denotes $\psi=0$.

Due to the correlation of $i$ and $\beta$, these fits do not further constrain the colatitude, but were used to refine the phase of best visibility $\phi_{1, I}=0.95, \phi_{1, R}=0.97$ and $\phi_{2}=0.42$. The phase shift of the primary spot in the $R$ and $I$-band with respect to $\phi=0$ determined from the $V$ band confirms the similar finding from the individual timings. Using the additional information about the phase of inferior conjunction, the azimuth of the spots can be determined. This was taken from blue-to-red zero crossing at HJD 24451247.5042 of the NaI absorption doublet, measured from Calar Alto spectroscopy which will be published later. According to our photometric ephemeris, this date corresponds to phase $\phi_{\text {phot }}=0.177$, and places the primary spot at $\psi_{1, I}=-80^{\circ}$ or $\psi_{1, R}=-70^{\circ}$ and the secondary spot at $\psi_{2}=90^{\circ}$.

All the above considerations neglect a possible extent and height of the accretion column, which are known to play a role at least in the better-studied systems. In HS 1023 the apparent location of the primary spot 
differs depending on the colour and thus cyclotron harmonic number. We regard this as a strong indication for a possible horizontal extent. Both vertical and horizontal extent would require a displacement to higher colatitudes in order to match the observed $\gamma$ values.

Additional information can be drawn from the beamed 3rd harmonic of the secondary spot. The observed doublehumped maxima occur when our line-of-sight is perpendicular to the magnetic field lines. For the case of non-inclined field lines this, marks the phase when the spot raises over the limb of the white dwarf and and gives a more unbiased measure for the duration of the faint phase. From the trailed spectrogram (Fig. 3) of Reimers et al. (1997) we find $\gamma=0.62$. The possible range of colatitudes widens accordingly to $90^{\circ} \lesssim \beta_{2} \lesssim 130^{\circ}$. The likely locations of the two accretion spots on the surface of the white dwarf in HS 1023 are illustrated in Fig. 6.

\section{Discussion}

Our photometric campaign provides further evidence that HS 1023 is in a persistent state of low accretion, and not just a normal AM Herculis binary coincidentally caught in low states during the previous observations. Contrary to HS 1023, the temporal reductions of the accretion rate in other polars occur only for about $50 \%$ of the time, as found from the sample of known polars in the ROSAT all sky survey (Beuermann \& Burwitz 1995) or the long-term light curve of AM Herculis itself (Hessman et al. 2000).

Secondaries in CVs are rapidly rotating, late-type stars and therefore expected to be magnetically very active. However, direct indicators like chromospheric X-ray emission or optical flares are easily outshone by accretion processes which are at least one order of magnitude brighter. The detection of a flare in HS 1023 is one of the few cases which show that the secondary is indeed magnetically active. Contrary to the giant flare $\left(E \sim 10^{34} \mathrm{erg}\right)$ measured during a low state of AM Her (Shakhovskoy et al. 1993), its energy of $10^{31} \mathrm{erg}$ is in the lower range of the energy distribution of flare stars. The fact that we find one such event within 10 hours of $B$-band observation agrees well with the flare frequency of well observed flare stars, e.g. YY Gem (Byrne 1995).

We determined the spectral type of secondary from broad-band colors to be $S p=4.5 \pm 1 \mathrm{M}$. This is just what is observed for CVs above and below the period gap in a comparative study of all CVs with known spectral type by Beuermann et al. (1998), lending further support to the idea that HS 1023 is a normal CV currently detached by the mechanisms that lead to the formation of the period gap. For the alternative hypothesis, that it is a pre-CV establishing contact within the period gap, the secondary should be in thermal equilibrium and have an earlier spectral type of M 3 if it is filling its Roche lobe. Correspondingly, a late spectral type of $S p=4.5$ as observed by us implies that it is underfilling its Roche lobe by $\sim 20 \%$.

The location of the accretion spots and thus the orientation of the magnetic field of the white dwarf in HS 1023 is quite peculiar compared with those of normal, high accretion rate polars. In a study of 11 AM Herculis systems, Cropper (1988) found that spot azimuths cluster in a narrow range of $\lesssim 90^{\circ}$ around a mean value $\psi \simeq 20^{\circ}$. As a direct consequence these systems must have i) a high degree of synchronism and ii) quite similar accretion and locking torques. The fact that the dipole in HS 1023 is orientated away by $\sim 90^{\circ}$ from this preferred configuration indicates either a possible asynchronism or that locking in case of a weak accretion torque leads to a different equilibrium configuration. Interestingly, the spot azimuths in HS 1023 agree within $20^{\circ}$ with the stable equilibrium points of $\psi=-90^{\circ}, 90^{\circ}$ for magnetostatic interaction (Joss et al. 1979).

HS 1023 is a prime target for a detailed study of the field structure and accretion processes of a weakly accreting white dwarf by phase-resolved, optical spectroscopy and polarimetry.

Acknowledgements. We thank our referee, Prof. Reimers, for helpful comments. This work was supported by the DFG under grant Schw536/1-1 and the DLR (former DARA GmbH) under grant and 50 OR 97068.

\section{References}

Byrne, P. B. 1995, Flares and Flashes, Proc. of IAU Coll. 151, Lect. Notes Phys. (Springer), 454

Beuermann, K., \& Burwitz, V. 1995, ASP Conf. Ser., 85, 95

Beuermann, K., Baraffe, I., Kolb, U., \& Weichhold, M. 1998, A\&A, 339, 518

Cropper, M. 1988, MNRAS, 231, 597

Hessman, F. V., Gänsicke, B. T., \& Mattei, J. A. 2000, A\&A, 237, 39

Joss, P. C., Katz, J. I., \& Rappaport, S. A. 1979, ApJ, 230, 176

Kirkpatrick, J. D., \& McCarthy, D. W. 1994, AJ, 107, 333

Mateo, M., \& Schechter, P. 1989, in 1st ESO/ST-ECF Data Analysis Workshop, ed. P. J. Grosbol, F. Murtagh, \& R. H. Warmels, 69

Moffett, T. J. 1974, ApJ, 273, 29

Reimers, D., Hagen, H.-J., \& Hopp, U. 1997, A\&A, 343, 157

Schwarzenberg-Czerny, A. 1989, MNRAS, 241, 153

Shakhovskoy, N. M., Alexeev, I. Yu., Andronow, I. L., \& Kolesnikov, S. V. 1992, Cataclysmic Variables and Related Physics, Ann. Israel Phys. Soci., 10, 237 